\title{
Abdominal wall reconstruction after decompressive laparotomy: a case utilizing tissue expanders, hybrid mesh and component separation
}

\begin{abstract}
Introduction: Abdominal compartment syndrome is a life threatening emergency. The abdominal wall can become obliterated in these patients who require decompression and can lead to ventral hernias in the long term. Abdominal wall reconstruction is now a multispecialty discipline involving general and plastic surgeons. We present a case of complex abdominal wall reconstruction using a novel hybrid mesh and component separation technique in a community hospital setting.
\end{abstract}

Case Presentation: A 43year old male underwent a decompressive laparotomy for abdominal compartment syndrome from necrotizing pancreatitis complicated with recurrent intra-abdominal abscess and splenic artery pseudoaneurysm. After a prolonged hospitalization, the patient underwent reconstruction with preoperative tissue expansion of the flank skin, bilateral component separation, and placement of Zenaprotm hybrid mesh. The patient developed a small segement of skin necrosis along his midline incision, which was resected and repaired. Overall, the patient reports an excellent quality of life and was also pleased with his cosmetic outcome.

Conclusion: Complex abdominal wall reconstruction can be successfully performed in a community hospital setting. The use of hybrid mesh in ventral hernia repairs can be a valuable tool in cases where high risk of contamination maybe present.

Keywords: component separation, tissue expanders, abdominal wall reconstruction
Volume 7 Issue 3 - 2017

\author{
Andrew McGregor,' Juila Toto, ${ }^{2}$ Daniel \\ Kleiner' \\ 'Danbury Hospital, Department of Surgery, USA \\ 2Danbury Hospital, Department of Surgery, Section of Plastic \\ Surgery, USA
}

Correspondence: Daniel Kleiner, Danbury Hospital, Department of Surgery, USA, Tel 203-739-7I 3I, Fax 203-7391554, Email andrew.mcgregor@wchn.org

Received: September 09, 2017 | Published: September I5, 2017

\section{Abbreviations: TFL, tensor fascia lata; ICG, indocyanine \\ Introduction}

Abdominal compartment syndrome is a life threatening emergency. It can cause multi system organ failure rapidly if not treated in a timely manner with abdominal decompression. The abdominal wall becomes obliterated in these patients who require decompression, and present a challenge to the general and plastic surgeon involved in the reconstructive process. The shift of abdominal wall reconstruction has now become a multi-specialty process including general and plastic surgeons. We discuss a case of a 43year old male who underwent a decompressive laparotomy with an acquired giant ventral hernia. He had a split thickness skin graft placed to his abdominal wall for temporary skin coverage. The reconstructive plan was to utilize tissue expanders to increase skin coverage and to use the hybrid mesh, Zenaprotm, with bilateral component separation to reconstruct his abdominal wall. This lead to a successful abdominal wall closure and decrease in the patient's morbidity.

\section{Case presentation}

We present a case of a 43year old male with acquired giant ventral wall hernia from a decompressive laparotomy for abdominal compartment syndrome from necrotizing pancreatitis. His past medical history was significant for alcohol abuse where he consumed 1 bottle of hard liquor per day. He had an extensive smoking history where he smoked 1-2 packs of cigarettes per day. His open abdomen was initially managed with a Vicryl mesh and negative pressure wound therapy. A $25 \times 30 \mathrm{~cm}$ split thickness skin graft was placed over the abdominal wall for temporary skin coverage. The rest of his hospitalization was complicated by multi drug resistant Pseudomonas $s p$. intra-abdominal abscesses and a splenic artery pseudoaneurysm. After embolization of his pseudoaneurysm and percutaneous drainage of the abscesses with aggressive antibiotic therapy including injection of Cefepime in to his Jackson Pratt drains and oral Metronidazole, he was discharged home after a 7 month hospitalization. He was continued on these same antibiotics for 2 months post operatively.

The reconstructive plan was coordinated with both a general and plastic surgeon. The operative plan was to insert flank tissue expanders to increase skin coverage and the patient's hernia was going to be repaired with a combination of component separation and hybrid mesh placement. The time frame for the final reconstruction would be one year after his discharge from the hospital.

Three main factors were involved in the success of this case. In regards to smoking cessation, the patient started on a nicotine patch while in the hospital before discharge and was able to be completely free from nicotine 2 months before the procedure. Preoperatively, it was stressed to the patient that the reconstruction would not proceed unless nicotine cessation was performed. Secondly, a weight loss protocol was undertaken. Preoperatively, the patient was given a goal to lose 40 pounds before surgery. His starting weight 4 months prior to his reconstruction was 227 pounds. His weight loss program was coordinated with a certified dietician in our practice. Meal management and exercise logs were created for the patient to account for his weight loss. His final weight was 211 pounds before surgery which was deemed acceptable for reconstruction; however, it did not meet the 40 pound goal. Nutritional markers were not used as a 
marker of correction for his nutritional status. Finally, tissue expander process was initiated after PMT tissue expanders were inserted into each flank six months prior to reconstruction. Tissue expansion occurred over 16weeks. An overall volume of $1325 \mathrm{ml}$ was instilled into the expanders with the patient undergoing fill sessions of $100 \mathrm{ml}$ per session. A preoperative CT scan was performed to demonstrate the abdominal wall defect (Figure 1).

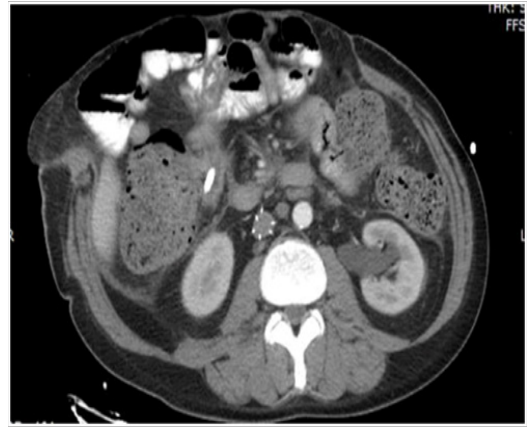

Figure I Preoperative CT scan demonstrating abdominal wall defect.

After appropriate tissue expansion, nicotine cessation, and weight loss the patient was brought to the operating room (Figure 2). The tissue expanders were removed and inspection of the abdomen revealed no further residual infection or abscess that could be identified. The hybrid mesh was sutured with 0 - Ethibon sutures in a figure of 8 fashion to the abdominal wall fascia circumfrentially (Figure 3). No quilting sutures were used. The component separation was then closed primarily with interrupted figure of eight sutures (Figure 4). A large amount of skin from the tissue expansion process was freely mobilized to the midline and closed primarily. No skin was trimmed or excised. Two Blake drains were placed in the subcutaneous tissues. The patient was taken to the PACU in stable condition. The patient was continued on Cefazolin postoperatively and discharged with Cephlexin. On postoperative day three, full thickness skin necrosis was noted. . The subcutaneous Blake drains were discontinued on the day of discharge. The necrosis was allowed to demarcate. 3 weeks later, the necrosis was excised and repaired. The patient was extremely pleased with his cosmetic outcome and continues to report a much better quality of life after this operation (Figure 5). ACT scan performed 6months later showed an intact repair (Figure 6). ${ }^{1-13}$

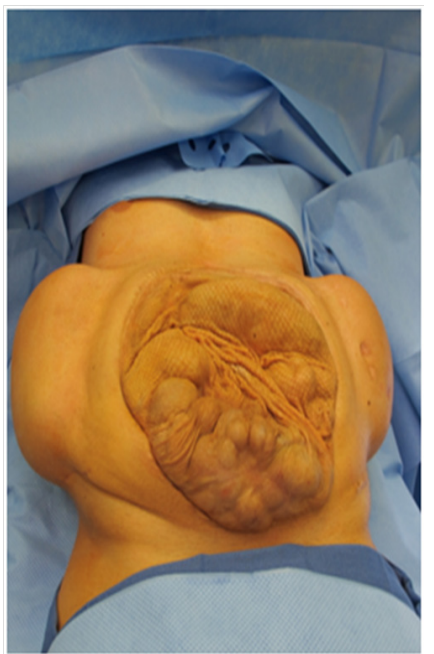

Figure 2 Preoperative Appearance abdominal wall skin graft and tissue expansion.

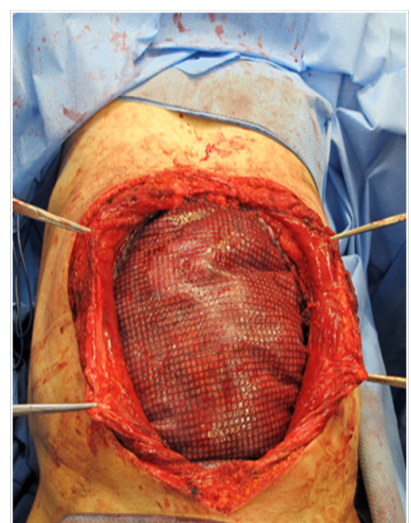

Figure 3 Retro-Rectus repair of ventral hernia defect with Zenapro Mesh.

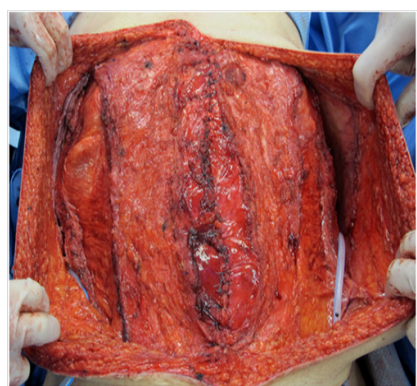

Figure 4 Closure of the component separation.

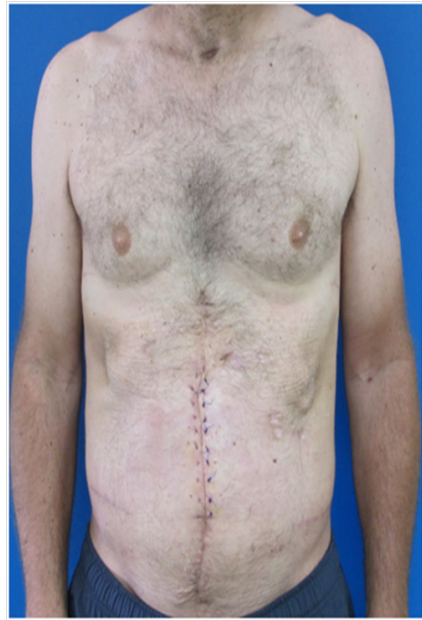

Figure 5 Post operative follow up appearance.

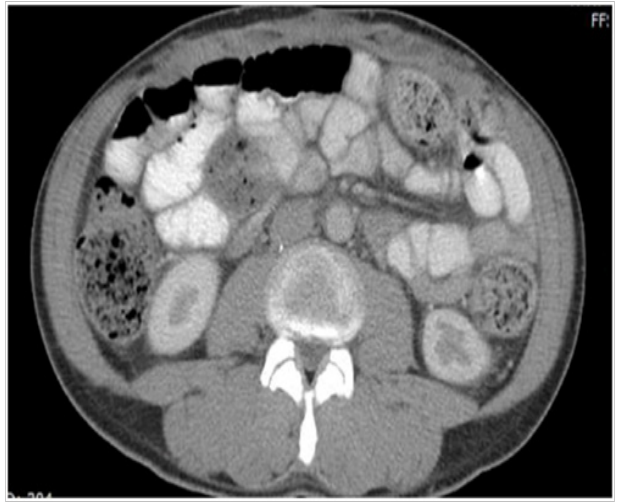

Figure 6 Month post operative CT scan demonstrating intact repair. 


\section{Acknowledgements}

None.

\section{Conflict of interests}

The authors have no financial or conflict of interests to declare.

\section{Informed patient consent}

Patient consent has been obtained for release of photos.

\section{References}

1. Criss CN, Petro CC, Krpata DM, et al. Functional abdominal wall reconstruction improves core physiology and quality-of-life. Surgery. 2014;156(1):176-182.

2. Poulose BK, Shelton J, Phillips S, et al. Epidemiology and cost of ventral hernia repair: making the case for hernia research. Hernia. 2012;16(2):179-183.

3. vanRamshorst GH, Eker HH, Hop WC, et al. Impact of incisional hernia on health-related quality of life and body image: a prospective cohort study. Am J Surg. 2012;204(2):144-150.

4. Ramirez O M, Ruas E, Dellon AL. Components separation method for closure of abdominal-wall defects: an anatomic and clinical study. Plast Reconstr Surg. 1990;86(3):519-526.
5. Slater NJ, van Goor H, Bleichrodt RP. Large and complex ventral hernia repair using components separation technique" without mesh results in a high recurrence rate. Am J Surg. 2015;209(1):170-179.

6. Fischer JP, Sieber B, Nelson JA, et al. A 15-year experience of complex scalp reconstruction using free tissue transfer-analysis of risk factors for complications. J Reconstr Microsurg. 2013;29(2):89-97.

7. Sue GR, Narayan D. Niche reconstructive techniques for complex abdominal wall reconstruction:a review. Am. 2014;80(4):327-334.

8. Carlson GW, Hester TR, Coleman JJ. The role of tensor fasciae latae musculocutaneous flap in abdominal wall reconstruction. Plast Surg Forum. 1988.

9. Darehzereshki A, Goldfarb M, Zehetner J, et al. Biologic versus nonbiologic mesh in ventral hernia repair: a systematic review and meta-analysis. World J Surg. 2014;38(1):40-50.

10. https://zenapro.cookmedical.com/

11. https://clinicaltrials.gov/ct2/show/NCT01784822

12. Holihan JL, Askenasy EP, Greenberg JA, et al. Component Separation vs. Bridged repair for large ventral hernias: a multi-institutional riskadjusted comparison, systematic review, and meta-analysis. Surg Infect (Larchmt). 2016;17(1):17-26.

13. Patel KM, Bhanot P, Franklin B, et al. Use of intraoperative indocyaningreen angiography to minimize wound healing complications in abdominal wall reconstruction. J Plast Surg Hand Surg. 2013;47(6):476-480. 